\title{
Direct-Acting Antiviral Therapy and Risk of Hepatocellular Carcinoma Recurrence in Patients with Chronic Hepatitis C
}

\author{
Chang Hun Lee and In Hee Kim \\ Department of Internal Medicine, Research Institute of Clinical Medicine of Chonbuk National University-Biomedical Research Institute \\ of Chonbuk National University Hospital, Chonbuk National University Medical School, Jeonju, Korea
}

\author{
Corresponding Author \\ In Hee Kim \\ ORCID https://orcid.org/0000-0003-3863-7907 \\ E-mail ihkimmd@jbnu.ac.kr
}

\author{
See "Independent Risk Factors for Hepatocellular Carcinoma Recurrence after Direct- \\ Acting Antiviral Therapy in Patients with Chronic Hepatitis C" by Young-Hwan Ahn, et al. \\ on page 410, Vol. 15, No. 3, 2021
}

Direct-acting antiviral (DAA) therapy for hepatitis C virus (HCV) infection showed high rates (>95\%) of sustained virologic response (SVR) with good safety. SVR after DAA therapy may result in improved liver dysfunction in patients with hepatocellular carcinoma (HCC), but its impact on the risk of HCC recurrence has been the subject of controversy. In this issue of Gut and Liver, Ahn et al. ${ }^{1}$ reported that $\mathrm{HCV}$-related $\mathrm{HCC}$ patients with complete response (CR) to treatment achieved acceptable SVR (88\%) with DAA therapy, and 37\% experienced HCC recurrence during the median follow-up period of 15.8 months (range, 4.4 to 29.9 months). Unfortunately, this study did not include a control group, which made it difficult to ascertain whether DAA therapy itself could increase or decrease the risk of HCC recurrence.

In 2016, Reig et al. ${ }^{2}$ sparked a debate by reporting an unexpectedly high HCC recurrence rate in HCV-infected patients following DAA therapy. Since then, studies evaluating the impact of DAA therapy on the risk of HCC recurrence after $\mathrm{CR}$ have produced conflicting data, and some studies have suggested a decreased risk of HCC recurrence, while others have shown the opposite results. A meta-analysis also showed that the use of DAA therapy was associated with a $>60 \%$ lower risk of HCC recurrence compared to patients without DAA treatment (odds ratio, 0.36; $95 \%$ confidence interval, 0.27 to $0.47 ; \mathrm{p}<0.001) .{ }^{3}$ However, most relevant studies had several limitations including heterogeneity between the cohorts in terms of stage of HCC, different HCC treatment options, multiple HCC treatments, the time interval between CR to HCC treatment and the ini- tiation of DAA therapy, surveillance protocols after DAA therapy, the duration of follow-up, and variability in the analytical methods used. Therefore, there are still no conclusive data that DAA therapy is associated with the risk of recurrent HCC in patients with CR to HCC treatment and the controversy continues.

Apart from these debates, it is evident that the risk of recurrent $\mathrm{HCC}$ continues in $\mathrm{HCV}$-infected patients with $\mathrm{CR}$ to HCC treatment after DAA therapy even achieving an SVR. The major risk factors for HCC recurrence are male gender, older age, non-SVR, advanced liver fibrosis, cirrhosis, and higher posttreatment alpha-fetoprotein levels. The time interval between curative therapy and DAA therapy has been suggested as a risk factor for HCC recurrence after HCC CR. ${ }^{4}$ Tsai et al. ${ }^{5}$ found that the recurrence rate was significantly higher among patients with a timespan of $<4$ months (54.6\%) between HCC treatment and DAA therapy compared to those with a timespan of $>4$ months (21.3\%, p=0.026). Singal et al. ${ }^{6}$ revealed that the risk of early recurrence could differ according to the initiation time of DAA therapy. The proportion of patients with HCC recurrence was $44.0 \%$ for those with a duration from HCC CR to DAA initiation of fewer than 3 months, $50.0 \%$ for those with a duration from HCC CR to DAA initiation of 4 to 6 months, and $36.9 \%$ for those with a duration of more than 6 months. However, these values did not achieve statistical significance. In multivariate analysis, Ogawa et al. ${ }^{7}$ reported that the time between previous HCC treatment and DAA exposure within 1 year (hazard ratio, 3.20; 95\% confidence interval, 1.29 to $9.65 ; \mathrm{p}=0.0011$ ) was signifi- 
cantly associated with HCC recurrence. In this issue of Gut and Liver, Ahn et al. ${ }^{1}$ also reported that a short last HCC treatment durability before DAA therapy ( $<12$ months) was an independent risk factor of HCC recurrence in both a multicenter Korean cohort and a nationwide cohort from the Korean Health Insurance Review and Assessment Service database. The authors defined last HCC treatment durability as the time between the final HCC treatment and the start of DAA therapy.

The delay of DAA therapy can provide a longer time for immune surveillance to work and allow a longer time to verify HCC CR, thereby minimizing the chance of misclassification bias. ${ }^{4}$ Meanwhile, SVR with DAA therapy can result in fibrosis regression and improvements in portal hypertension and liver dysfunction, which is the major cause of mortality in patients with HCC CR and untreated HCV infections. Singal et al. ${ }^{8}$ reported that patients with prior HCC had a median time from HCC CR to DAA initiation of 7.7 months, and DAA therapy was associated with a significant reduction in the risk of death (hazard ratio, $0.54 ; 95 \%$ confidence interval, 0.33 to 0.90 ). In a systematic review with a meta-analysis, Saraiya et al. ${ }^{9}$ suggested an acceptable HCC recurrence rate after DAA therapy that was delayed at least 6 months after the HCC CR. However, the appropriate timing of DAA initiation after CR in $\mathrm{HCV}$ related HCC patients remains controversial. The American Gastroenterological Association expert review suggested that DAA therapy should not be withheld from patients with CR to HCC therapy, but DAA therapy can be deferred 4 to 6 months to confirm response to HCC therapy. ${ }^{10}$ However, even after SVR with DAA therapy in patients with HCC CR, clinicians and patients should be vigilant for $\mathrm{HCC}$ recurrence and require regular surveillance.

\section{CONFLICTS OF INTEREST}

No potential conflict of interest relevant to this article was reported.

\section{ORCID}

Chang Hun Lee

https://orcid.org/0000-0001-7244-8849

In Hee Kim

\section{REFERENCES}

1. Ahn YH, Lee H, Kim DY, et al. Independent risk factors for hepatocellular carcinoma recurrence after direct-acting antiviral therapy in patients with chronic hepatitis C. Gut Liver 2021;15:410-419.

2. Reig M, Mariño Z, Perelló C, et al. Unexpected high rate of early tumor recurrence in patients with HCV-related HCC undergoing interferon-free therapy. J Hepatol 2016;65:719726.

3. Lui FH, Moosvi Z, Patel A, et al. Decreased risk of hepatocellular carcinoma recurrence with direct-acting antivirals compared with no treatment for hepatitis C: a meta-analysis. Ann Gastroenterol 2020;33:293-298.

4. Gao X, Zhan M, Wang L, Ding Y, Niu J. Timing of DAA initiation after curative treatment and its relationship with the recurrence of HCV-related HCC. J Hepatocell Carcinoma 2020;7:347-360.

5. Tsai PC, Huang CF, Yu ML. Unexpected early tumor recurrence in patients with hepatitis $\mathrm{C}$ virus-related hepatocellular carcinoma undergoing interferon-free therapy: issue of the interval between HCC treatment and antiviral therapy. J Hepatol 2017;66:464.

6. Singal AG, Rich NE, Mehta N, et al. Direct-acting antiviral therapy not associated with recurrence of hepatocellular carcinoma in a Multicenter North American Cohort Study. Gastroenterology 2019;156:1683-1692.

7. Ogawa E, Furusyo N, Nomura H, et al. Short-term risk of hepatocellular carcinoma after hepatitis $\mathrm{C}$ virus eradication following direct-acting anti-viral treatment. Aliment Pharmacol Ther 2018;47:104-113.

8. Singal AG, Rich NE, Mehta N, et al. Direct-acting antiviral therapy for hepatitis $\mathrm{C}$ virus infection is associated with increased survival in patients with a history of hepatocellular carcinoma. Gastroenterology 2019;157:1253-1263.

9. Saraiya N, Yopp AC, Rich NE, Odewole M, Parikh ND, Singal AG. Systematic review with meta-analysis: recurrence of hepatocellular carcinoma following direct-acting antiviral therapy. Aliment Pharmacol Ther 2018;48:127-137.

10. Singal AG, Lim JK, Kanwal F. AGA clinical practice update on interaction between oral direct-acting antivirals for chronic hepatitis $\mathrm{C}$ infection and hepatocellular carcinoma: expert review. Gastroenterology 2019;156:2149-2157. 